\title{
Chlorpromazine-induced perturbations of bile acids and free fatty acids in cholestatic liver injury prevented by the Chinese herbal compound Yin-Chen-Hao-Tang
}

Qiaoling Yang ${ }^{1 \dagger}$, Fan Yang $^{1 \dagger}$, Xiaowen Tang ${ }^{1}$, Lili Ding ${ }^{1}$, Ying Xu', Yinhua Xiong ${ }^{1}$, Zhengtao Wang ${ }^{1}$ and Li Yang ${ }^{1,2,3^{*}}$

\begin{abstract}
Backgrounds: Yin-Chen-Hao-Tang (YCHT), a commonly used as a traditional chinese medicine for liver disease. Several studies indicated that YCHT may improving hepatic triglyceride metabolism and anti-apoptotic response as well as decreasing oxidative stress. However, little is known about the role of YCHT in chlorpromazine (CPZ) -induced chlolestatic liver injury. Therefore, we aimed to facilitate the understanding of the pathogenesis of cholestatic liver injury and evaluate the effect of Yin-Chen-Hao-Tang (YCHT) on chlorpromazine (CPZ)-induced cholestatic liver injury in rats based on the change of bile acids (BAs) and free fatty acids (FFAs) alone with the biochemical indicators and histological examination.

Methods: We conducted an experiment on CPZ-induced cholestatic liver injury in Wistar rats with and without YCHT for nine consecutive days. Serum levels of alanine aminotransferase (ALT), aspartate aminotransferase (AST), albumin (ALB), total bilirubin (TBIL), total cholesterol (TC), triglycerides (TG), low density lipoprotein-cholesterol $(\mathrm{LDL}-\mathrm{C})$ were measured to evaluate the protective effect of YCHT against chlorpromazine (CPZ)-induced cholestatic liver injury. Histopathology of the liver tissue showed that pathological injuries were relieved after YCHT pretreatment. In addition, ultra-performance lipid chromatography coupled with quadrupole mass spectrometry (UPLC-MS) and gas chromatography coupled with mass spectrometry (GC-MS) was applied to determine the content of bile acids, free fatty acids, respectively.

Results: Obtained data showed that YCHT attenuated the effect of CPZ-induced cholestatic liver injury, which was manifested by the serum biochemical parameters and histopathology of the liver tissue. YCHT regulated the lipid levels as indicated by the reversed serum levels of TC, TG, and LDL-C. YCHT also regulated the disorder of BA and FFA metabolism by CPZ induction.

(Continued on next page)
\end{abstract}

\footnotetext{
* Correspondence: yangli7951@hotmail.com

${ }^{\dagger}$ Equal contributors

${ }^{1}$ The Ministry of Education Key Laboratory for Standardization of Chinese Medicines and the State Administration of TCM (SATCM) Key Laboratory for New Resources and Quality Evaluation of Chinese Medicines, Institute of Traditional Chinese Materia Medica, Shanghai University of Traditional Chinese Medicine, 201210 Shanghai, China

${ }^{2}$ Center for Chinese Medical Therapy and Systems Biology, Shanghai University of Traditional Chinese Medicine, Shanghai 201203, China Full list of author information is available at the end of the article
} 
(Continued from previous page)

Conclusions: Results indicated that YCHT exerted a protective effect on CPZ-induced cholestasis liver injury. The variance of BA and FFA concentrations can be used to evaluate the cholestatic liver injury caused by CPZ and the hepatoprotective effect of YCHT.

Keywords: Bile acids, Free fatty acids, Chlorpromazine, Yin-Chen-Hao-Tang (YCHT), UPLC-MS, Hepatoprotective effect

\section{Background}

Cholestasis is a prevalent form of chronic liver disease characterized as a consequence of disturbed hepatocellular secretion of bile, impaired bile formation, and slow bile flow [1]. Chlorpromazine (CPZ), a member of the largest class of first-generation phenothiazine antipsychotic drugs, is a primary drug in psychiatric treatment [2]. The hepatoxicity of CPZ should not be ignored during its therapeutic use [3]. CPZ-induced hepatotoxicity may be associated with the mechanism involving sustained activation of JNK, which leads to inflammation $[4,5]$. In addition, CPZ can induce cholestasis by inhibiting bile flow in vivo [6]. Previous studies on CPZinduced intrahepatic cholestasis in vitro demonstrated that the mechanismis associated with the alteration of bile acid (BA) transport receptors and oxidative stress by altering mitochondrial membrane potential and the pericanalicular distribution of F-actin [7]. Considerable amount of evidence indicates that $\mathrm{CPZ}$ can be used as an excellent model of drug-induced liver injury and is usually administered to mimic drug-induced cholestasis [8-10]. However, the diagnosis and assessment of the initial toxic effects of CPZ are limited and do not accurately predict cholestasis.

The detergent character of BAs exerts an important role in regulating liver metabolism [11]. Cholestasis is an impairment or cessation of bile flow. Cholestasis leads to hepatic and systemic accumulation of potentially toxic biliary compounds, such as BAs and bilirubin, resulting in oxidative stress, apoptosis, and subsequent damage to the liver parenchyma [12]. Several studies report that the disruption of BA homeostasis is closely related to hepatic dysfunction [13-16] and intestinal ailments [17,18]. Free fatty acid (FFA) is an energy provider that plays an important role in control energy metabolism and glucose metabolism. However, FFAs can lead to cell injury and apoptosis and are key mediators of lipotoxicity within hepatocytes $[19,20]$. Studies indicate that abnormal FFA metabolism is associated with liver disease [21,22]. Therefore, maintaining the metabolism of BA and FFA is important for liver metabolism function. In our previous study, the validated ultra-performance lipid chromatography coupled with quadrupole mass spectrometry (UPLC-MS) method based on BA and gas chromatography coupled with mass spectrometry (GC-MS) based on FFA were applied to evaluate the carbon tetrachloride, $\alpha$ naphthylisothiocyanate (ANIT) and acetaminophen-induced liver injury in rats [23,24].

YCHT is a famous and classic Chinese herbal compound that consists of three medicinal materials, namely, Artemisia capillaris Thunb (Tarragon), Gardenia jasminoides Ellis (Gardenia), and Rheum officinale Baill (Rhubarb). YCHT is recorded in "Shang Han Lun" and has been used to treat jaundice for more than a thousand years. YCHT is considered as a hepatoprotective agent by improving hepatic triglyceride metabolism and antiapoptotic response as well as decreasing oxidative stress [25-30]. Related proteomics data suggest that the therapeutic effects of YCHT may be associated with the regulation of lipid biosynthesis [31]. Limited data are available about the efficacy of YCHT on CPZ-induced cholestasis and its corresponding mechanism.

This study aimed to evaluate the protective effect of YCHT on CPZ-induced cholestatic liver injury based on the variations of endogenous metabolites and provide insights into the role of BAs and FFAs in the progression of the pathological changes.

\section{Methods}

\section{Chemicals and reagents}

Rhubarb was provided by Shanghai Hutchison Pharmaceuticals (batch number: 121012; Shanghai, China). Gardenia and Tarragon were purchased from Shanghai Cambridge Traditional Chinese Medicine decoction pieces company (batch number: 081226; Shanghai, China) and Bozhou (batch number: 20100708; Anhui, China) medicine market, respectively. They were authenticated as Rheum officinale Baill, Gardenia jasminoides Ellis, Artemisia capillaris Thunb by Dr. LiHong Wu (Professor, Instituent of Chinese Materia Medica, Shanghai University of Tradational Chinese Medicine). The voucher specimens (dh-121012, ych-20100708, zz-081226) were deposited in the Herbarium of Instituent of Chinese Materia Medica, Shanghai University of Traditional Chinese Medicine. CPZ hydrochloride injection was purchased from Shanghai Harvest Pharmaceutical Co., Ltd. $\alpha$-muricholic acid $(\alpha-\mathrm{MCA}), \beta$-muricholic acid ( $\beta$-MCA), $\omega$-muricholic acid ( $\omega$-MCA), Cholic acid (CA), deoxycholic acid (DCA), chenocholic acid (CDCA), lithocholic acid, ursodeoxycholic acid, hyodesoxycholic acid, 
glycocholic acid (GCA), taurocholic acid (TCA), glycodeoxycholic acid (GDCA), taurodeoxycholic acid (TDCA), glycochenodeoxycholic acid (GCDCA), taurochenodeoxycholic acid (TCDCA), glycoursodeoxycholic acid, taurohyodesoxycholic acid (THDCA), glycolithocholic acid (GLCA), taurohyodesoxycholic acid (TLCA), lauric acid (C12:0), tetradecanoic acid (C14:0), palnitic acid (C16:0), heptadecanoic acid (C17:0), stearic acid (C18:0), arachidic acid (C20:0), docosanoic acid (C22:0), lignoceric acid (C24:0), palmitoleic acid (C16:1n7), oleic acid (C18:1n9), vaccenic acid (C18:1n7), linoleic acid (C18:2n6), $\gamma$-linolenic acid (C18:3n6), linolenic acid (C18:3n3), eicosatrienoic acid (C20:3n6), arachidonic acid (C20:4n6), eicosapentaenoic acid (C20:5n3), and docosahexaenoic acid (C22:6n3) were purchased from Sigma-Aldrich. Their purities were above 98\%. Acetonitrile, methanol, formic acid, and ammonium acetate (HPLC grade) were purchased from Fisher Scientific (Nepean, Ontario, Canada). De-ionized water was prepared by Milli-Q system (Millipore, Bedford, MA). The other solvents were of analytical grade and obtained from Shanghai Chemical Factory (Shanghai, China).

\section{Preparation of YCHT and chemical analysis by UPLC- QTOF/MS/MS}

YCHT was extracted as follows. Crude drug materials of Rhubarb (30 g), Gardenia (45 g), and Artemisia capillaries $(90 \mathrm{~g})$ were decocted three times in boiling water $(3000 \mathrm{~mL})$ for $1.5 \mathrm{~h}$ each time. The decoctions were filtered, combined, and concentrated to the volume of $300 \mathrm{~mL}$. Liquid chromatography/electrospray ionization time-of-flight mass spectrometry (LC/ESI-TOF MS) was adopted to validate the chemical composition of the aqueous extract of YCHT. Samples were separated on the Waters ACQUITY BEH C18 column $(100 * 2.1 \mathrm{~mm}$, $1.7 \mu \mathrm{m})$ with the column temperature maintained at $45^{\circ} \mathrm{C}$. The mobile phase consisted of $0.1 \%$ formic acid in $5 \mathrm{mM}$ ammonium acetate aqueous solution (A) and methanol (B) at a flow rate of $0.3 \mathrm{~mL} / \mathrm{min}$. The elution gradient was performed as follows. During the first $1 \mathrm{~min}$, the eluent composition was set at $95 \% \mathrm{~A}$ and $5 \% \mathrm{~B}$, which was linearly changed to $75 \% \mathrm{~A}$ and $25 \% \mathrm{~B}$ in $4 \mathrm{~min}$, and then the proportion of $\mathrm{B}$ was increased to $50 \%$ in the next $20 \mathrm{~min}$. The proportion of B was linearly increased by $95 \%$ in the next $6 \mathrm{~min}$. The sample injection volume was $5 \mu \mathrm{L}$. Mass spectrometry was performed on the Waters SYNAPT QTOF/MS (Waters Corp.). The mass range was set at m/z $100 \mathrm{Da}$ to $1200 \mathrm{Da}$. The MS/MS experiments were performed at variable collision energy $(20 \mathrm{eV}$ to $30 \mathrm{eV}$ ). The data were processed using MassLynx 4.1 software (Waters Corp.).

\section{Ethics statement}

The Guide for the Care and Use of Laboratory Animals was strictly complied, and the animal experiment protocols were approved by the Institutional Animal Committee of Shanghai University of Traditional Chinese Medicine [Permit number: SCXK (Hu) 2012-0002]. All surgical procedures were performed under ether, and all efforts were made to reduce animal suffering.

\section{Animal administration and sample collection}

Male Wistar rats (220 $\pm 20 \mathrm{~g}, 6$ weeks to 8 weeks of age) were obtained from the laboratory animal center of Shanghai University of Traditional Chinese Medicine (SHUTCM, Shanghai). The animals were maintained on a 12/12 h light-dark cycle (lights on at 7:00 am) with regulated temperature and humidity. During the whole experimental process, rats were fed with certified standard rat chow and tap water ad libitum. All rats were allowed to acclimatize for 7 days before experimentation and randomly divided into three groups (eight rats for each group). Group 1 served as non-treated controls, and group 2 served as CPZ-treated model group. The rats of group 3 were intragastrically given with YCHT, which was suspended in distilled water at doses of $8 \mathrm{~g} / \mathrm{kg}$ $(10 \mathrm{~mL} / \mathrm{kg}, \mathrm{B} . \mathrm{W}$.) every $24 \mathrm{~h}$ for nine consecutive days. At $12 \mathrm{~h}$ after administration of the seventh dose, the rats of groups 2 and 3 received CPZ by intraperitoneal injection at a dose of $75 \mathrm{mg} / \mathrm{kg}(3.6 \mathrm{~mL} / \mathrm{kg}$, B.W.), which is well documented to induce liver injury and cholestasis. Meanwhile, group 1 received intragastrical treatment of physiological saline in an equal volume as for groups 2 and 3 $(5 \mathrm{~mL} / \mathrm{kg} \mathrm{B.W.}$.). At the end of the study, all rats were euthanized by $\mathrm{CO}_{2}$ inhalation in a 12-h fasting state. Retroorbital blood samples were collected into tubes at $48 \mathrm{~h}$ after the last treatment and then immediately centrifuged at $4^{\circ} \mathrm{C}$ for $10 \mathrm{~min}(3000 \mathrm{~g})$ to separate the serum. The resulting serum samples were stored at $-80^{\circ} \mathrm{C}$ until analysis. Each liver sample was isolated and stored at $-80^{\circ} \mathrm{C}$ for further analysis, except for the central part of the right large lobe, which was used for histological examination.

\section{Biochemical determination and histological examination}

The collected blood samples were placed at room temperature for $4 \mathrm{~h}$ and centrifuged at $13000 \mathrm{~g}$ for $10 \mathrm{~min}$ at $4^{\circ} \mathrm{C}$ to obtain serum. The serum contents of ALT, AST, ALB, TBIL, TC, TG, and LDL-C were determined using a commercially available clinical test kit and a chemistry analyzer system (HITACHI 7080; Japan). The liver samples obtained from the central part of the right large lobe were fixed with $10 \%$ formalin in PBS for $24 \mathrm{~h}$ and then washed with tap water, dehydrated in alcohol, and embedded in paraffin. The $4 \mu \mathrm{m}$ thick sections were obtained, deparaffinized, dehydrated in ethanol (50\% to 100\%), and cleared with xylene. Each slide was stained with hematoxylin and eosin, and then histological assessment was performed by Shanghai Showbio Biotech, Inc. 


\section{Quantitative determination of BAs}

The BA quantification method [23] was conducted with modification. The BA mix reference standards were prepared by dissolving each BA in methanol. BA was extracted in serum in serum as follows. In brief, $300 \mu \mathrm{L}$ of methanol was added to $100 \mu \mathrm{L}$ of serum, and the mixture was vortexed for $2 \mathrm{~min}$ and centrifuged $\left(20000 \mathrm{~g}, 4^{\circ} \mathrm{C}\right)$ for $10 \mathrm{~min}$. The supernatant was separated and evaporated to dryness, and the residue was stored at $-20^{\circ} \mathrm{C}$ and reconstituted in $100 \mu \mathrm{L}$ of methanol-water (55:45; containing a mixture of $5 \mathrm{mM}$ ammonium acetate and $0.1 \%$ formic acid) before analysis. The sample solution was centrifuged at $20000 \mathrm{~g}$ for $10 \mathrm{~min}$ at $4^{\circ} \mathrm{C}$, and a $5 \mu \mathrm{L}$ of aliquot was injected for UPLC-MS analysis.

BAs were determined using the Waters ACQUITY ultra-performance lipid chromatograph system (Waters, MA, USA) equipped with the Acquity UPLC BEH C18 $\left(1.7 \mu \mathrm{m}, 2.1^{*} 100 \mathrm{~mm}\right.$, Waters) column with a temperature of $45^{\circ} \mathrm{C}$. The mobile phase consisted of $0.1 \%$ formic acid in $5 \mathrm{mM}$ ammonium acetate aqueous solution (A) and methanol (B) at a flow rate of $0.3 \mathrm{~mL} / \mathrm{min}$. The elution gradient was performed as follows. During the first $1 \mathrm{~min}$, eluent composition was set at 55\% A and 45\% B, which was linearly changed to $62 \% \mathrm{~A}$ and $38 \% \mathrm{~B}$ in $2.6 \mathrm{~min}$, and then the proportion of B was increased to $80 \%$ in the next $8.8 \mathrm{~min}$. The sample injection volume was $5 \mu \mathrm{L}$.

MS analysis was performed using ZQ 2000 quadrupole spectrometry equipped with an ESI probe operated with Selective Ion Monitoring (SIM) in the negative-ion mode
(Waters, MA, USA). The capillary and cone voltages were set at 3.0 and $55 \mathrm{~V}$, respectively. The source temperature was $120^{\circ} \mathrm{C}$, and the desolvation temperature was $300^{\circ} \mathrm{C}$. The desolvation gas flow was set at $700 \mathrm{~L} / \mathrm{h}$, and the cone gas flow rate was set at $50 \mathrm{~L} / \mathrm{h}$. Data were acquired and processed using MassLynx 4.1 software.

\section{Quantitative determination of FFAs}

The quantification method [24] was conducted with modification. A mixed standard solution of fatty acid methyl esters was prepared in $5 \% \mathrm{H}_{2} \mathrm{SO}_{4}-\mathrm{CH}_{3} \mathrm{OH}$. The FFAs in serum were extracted. Twenty microliters of $1000 \mu \mathrm{g} / \mathrm{mL}$ mixed internal standard (C19:2n10 and its methyl ester) was added to $100 \mu \mathrm{L}$ of serum. FFAs were methylated in $5 \% \mathrm{H}_{2} \mathrm{SO}_{4}-\mathrm{CH}_{3} \mathrm{OH}$. Lipid extraction was performed using $n$-hexane. The $n$-hexane phase was collected, evaporated to dryness in the $\mathrm{N}_{2}$ atmosphere, and re-dissolved by $500 \mu \mathrm{L}$ of $n$-hexane.

Experiments were performed on a 6800 GC system (Agilent Technologies, Santa Clara, CA, USA) coupled with a 5973 mass spectrometer. The GC system was equipped with a 7683B series injector. The chromatographic separation was performed with a DB-225 MS capillary column $(60 \mathrm{~m} * 0.25 \mathrm{~mm}$ i.d., $0.25 \mu \mathrm{m}$ film thickness, Agilent, Folsom, CA, USA). The oven gradient temperature was performed as follows. During the initial $1 \mathrm{~min}$, the temperature was set at $70^{\circ} \mathrm{C}$, increased to $200^{\circ} \mathrm{C}$ by $40^{\circ} \mathrm{C} / \mathrm{min}$ in the next $20 \mathrm{~min}$, changed to $230^{\circ} \mathrm{C}$ by a second gradient of $5^{\circ} \mathrm{C} / \mathrm{min}$, and held for $25 \mathrm{~min}$. A 5973

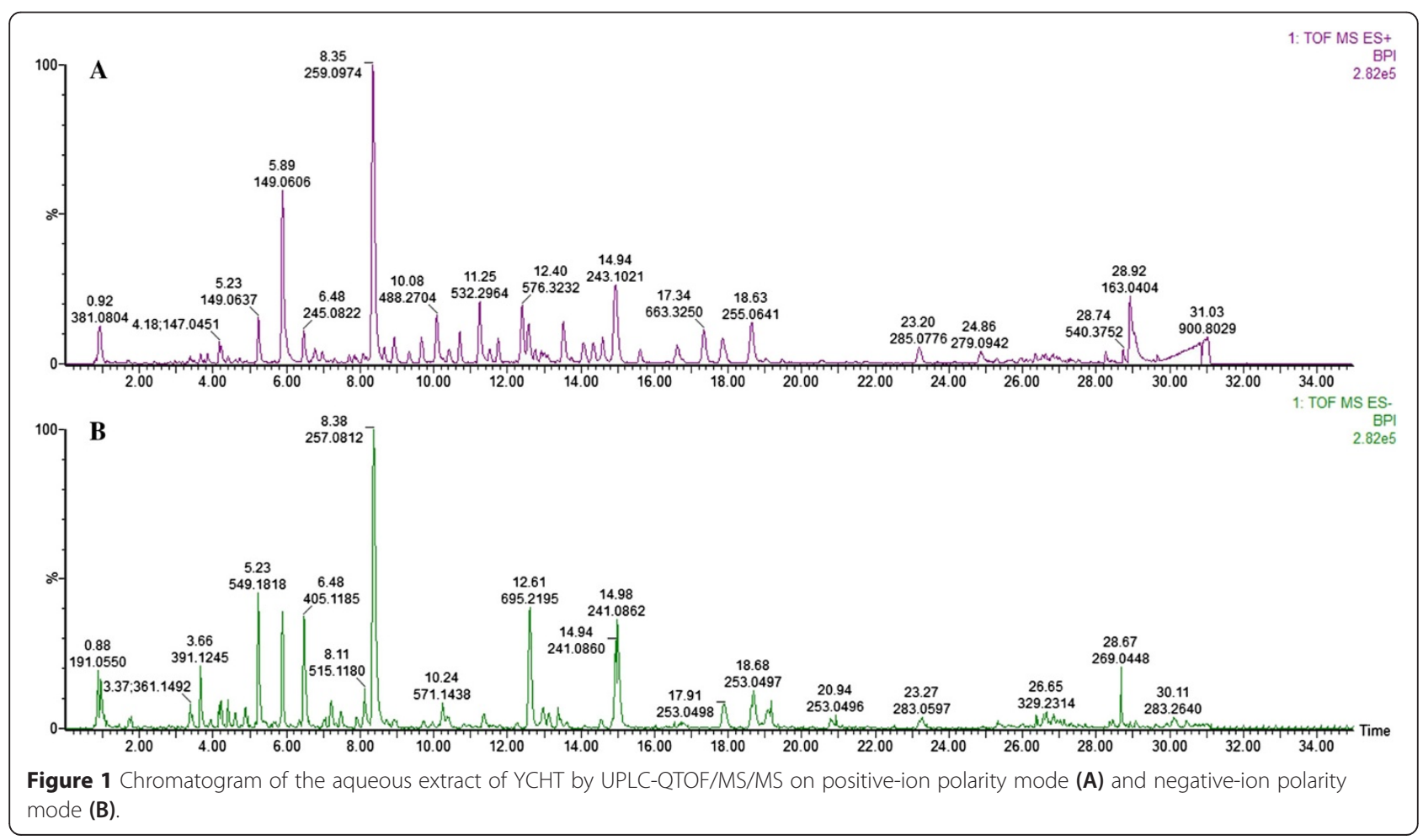


mass spectrometer in the electron impact was operated at $70 \mathrm{eV}$ on the SIM mode. The temperatures of the ion source and quadrupole were adjusted to 230 and $150^{\circ} \mathrm{C}$, respectively.

\section{Data processing and statistical analysis}

UPLC-MS and GC-MS data were acquired and processed using MassLynx 4.1 and enhanced MSD ChemStation software (Agilent Technologies, Inc., USA), respectively. Statistics was analyzed with one-way ANOVA and the least significant difference test (SPSS 18.0 software, Inc., Chicago, USA). The difference was considered statistically significant when $p \leq 0.05$, very significant when $p \leq 0.01$, and highly significant when $p \leq 0.001$. Multivariate statistical analysis was conducted by SIMCA-P 11.5 (Umetrics, Umea, Sweden).

\section{Results}

Chemical analysis of YCHT

The aqueous extract of YCHT was analyzed by Waters SYNAPT G2 QTOF/MS. The UPLC-QTOF/MS chromatogram is shown in Figure 1. The separated compounds were clarified by comparing the Rt values and the MS characteristics in both positive- and negative-ion polarity modes (see Table 1 ).

YCHT reversed the alterations of serum biochemicals in rats with $\mathrm{CPZ}$-induced cholestatic liver injury

Several clinical parameters in the serum were measured to monitor the toxic effects of CPZ and confirm the occurrence of cholestatic liver injury induced by $\mathrm{CPZ}$ in the animal model. Alone administration of CPZ induced a significant increase in serum level of ALT, AST, ALB, TBIL in rats as compared to normal control group, suggested that $\mathrm{CPZ}$ exposure has successfully lead to cholestatic liver injury. Other clinical parameters measured in serum were also significantly changed. TC, TG, and LDL-C were significantly increased in the model group compared with those in the control group, which indicates that CPZ exposure may affect lipid metabolism. However, the group pretreated with YCHT significantly declined the CPZ-induced elevation in the serum levels of ALT, AST, ALB, TBIL, TC, TG, and LDL-C, and concentration of TC (Figure 2 and Table 2). No adverse health effects on rats were observed during the experiment.

\section{Effect of YCHT on histological changes}

Main changes, such as proliferation of bile duct, expansion of hepatic sinus, necrosis of hepatocyte, and effusion of inflammation factors, were observed in CPZ-stimulated hepatotoxicity animal models (Figure 3B). However, these changes were suppressed in the liver sections of rats pretreated with YCHT. This finding indicates mild necrosis of hepatocyte and effusion of inflammation factors as shown
Table 1 Main compounds in the aqueous extract of YCHT by UPLC-QTOF/MS/MS

\begin{tabular}{|c|c|c|c|}
\hline Peaks & $\begin{array}{l}\text { Retention } \\
\text { time (min) }\end{array}$ & $M / Z$ & Identified compounds \\
\hline 1 & 0.84 & $181.0715[\mathrm{M}-\mathrm{H}]^{-}$ & Mannitol \\
\hline 2 & 1.79 & $169.0135[\mathrm{M}-\mathrm{H}]^{-}$ & Gallic acid \\
\hline 3 & 3.63 & $373.1123[\mathrm{M}-\mathrm{H}]^{-}$ & Geniposidic acid \\
\hline 4 & 3.66 & $391.1245[\mathrm{M}-\mathrm{H}]^{-}$ & Gardenoside \\
\hline 5 & 3.93 & $137.023[\mathrm{M}-\mathrm{H}]^{-}$ & 3,4-dihydroxybenzaldehyde \\
\hline 6 & 4.34 & $353.0870[\mathrm{M}-\mathrm{H}]^{-}$ & Chlorogenic acid \\
\hline 7 & 4.35 & $153.0192[\mathrm{M}-\mathrm{H}]^{-}$ & 3,4-dihydroxybenzoic acid \\
\hline 8 & 4.83 & $515.1190[\mathrm{M}-\mathrm{H}]^{-}$ & 1,3-dicaffeoylquinic acid \\
\hline 9 & 5.23 & $549.1811[\mathrm{M}-\mathrm{H}]^{-}$ & Genipin-1- $\beta$-D-gentiobioside \\
\hline 10 & 5.66 & $135.0446[\mathrm{M}-\mathrm{H}]^{-}$ & 4-hydroxyacetophenone \\
\hline 11 & 5.88 & $387.1285[\mathrm{M}-\mathrm{H}]^{-}$ & Geniposide \\
\hline 12 & 5.88 & $225.0766[\mathrm{M}-\mathrm{H}]^{-}$ & Genipin \\
\hline 13 & 7.21 & $477.1404[\mathrm{M}-\mathrm{H}]^{-}$ & Isolindleyin \\
\hline 14 & 7.47 & $183.102[\mathrm{M}-\mathrm{H}]^{-}$ & Jasminodiol \\
\hline 15 & 7.63 & $463.0869[\mathrm{M}-\mathrm{H}]^{-}$ & Isoquercitrin \\
\hline 16 & 8.11 & $515.1196[\mathrm{M}-\mathrm{H}]^{-}$ & 3,5-dicaffeoylquinic acid \\
\hline 17 & 8.31 & $207.0659[\mathrm{M}+\mathrm{H}]^{+}$ & 7-dimethylesculetin \\
\hline 18 & 8.32 & $419.1357[\mathrm{M}-\mathrm{H}]^{-}$ & Poniticin \\
\hline 19 & 8.35 & $445.0760[\mathrm{M}-\mathrm{H}]^{-}$ & Rhein-1-O- $\beta$-D-glucopyranoside \\
\hline 20 & 10.37 & $515.1189[\mathrm{M}-\mathrm{H}]^{-}$ & 4,5-dicaffeoylquinic acid \\
\hline 21 & 12.6 & $695.1295[\mathrm{M}-\mathrm{H}]^{-}$ & $\begin{array}{l}\text { 6-O-trans- } \\
\text { coumaroylgenipin-gentiobioside }\end{array}$ \\
\hline 22 & 12.97 & $755.2415[\mathrm{M}-\mathrm{H}]$ & $\begin{array}{l}\text { 6-O-trans-sinapolygenipin- } \\
\text { gentiobioside }\end{array}$ \\
\hline 23 & 14.86 & $593.1865[\mathrm{M}-\mathrm{H}]^{-}$ & 6-O-sinapolygeniposide \\
\hline 24 & 23.27 & $283.0759[\mathrm{M}-\mathrm{H}]^{-}$ & Physcion \\
\hline 25 & 25.35 & $283.0247[\mathrm{M}-\mathrm{H}]^{-}$ & Rhein \\
\hline 26 & 25.47 & $239.0343[\mathrm{M}-\mathrm{H}]^{-}$ & Alizarin \\
\hline 27 & 28.76 & $269.0451[\mathrm{M}-\mathrm{H}]^{-}$ & Emodin \\
\hline 28 & 29.12 & $169.0135[\mathrm{M}-\mathrm{H}]^{-}$ & Chrysophanol \\
\hline
\end{tabular}

in Figure 3C.In addition, the necrosis of hepatocyte was confirmed by the quantitative scoring (Figure 3D).

\section{YCHT affects the serum BA and FFA profiles in rats with} CPZ-induced cholestatic liver injury

The characterization and quantification of BAs and FFAs in serum are focused in the study of metabolic progress. Alterations in BA and FFA profiles are observed in nutritional diseases, metabolic disorders, obesity, cancer, and gastrointestinal diseases. Thus, the optimized reversed-phase UPLC-MS and GC-MS conditions were applied to determine the contents of individual BA and FFA in serum and further interpret the biological events. The data based on the quantitative analysis of BAs and 


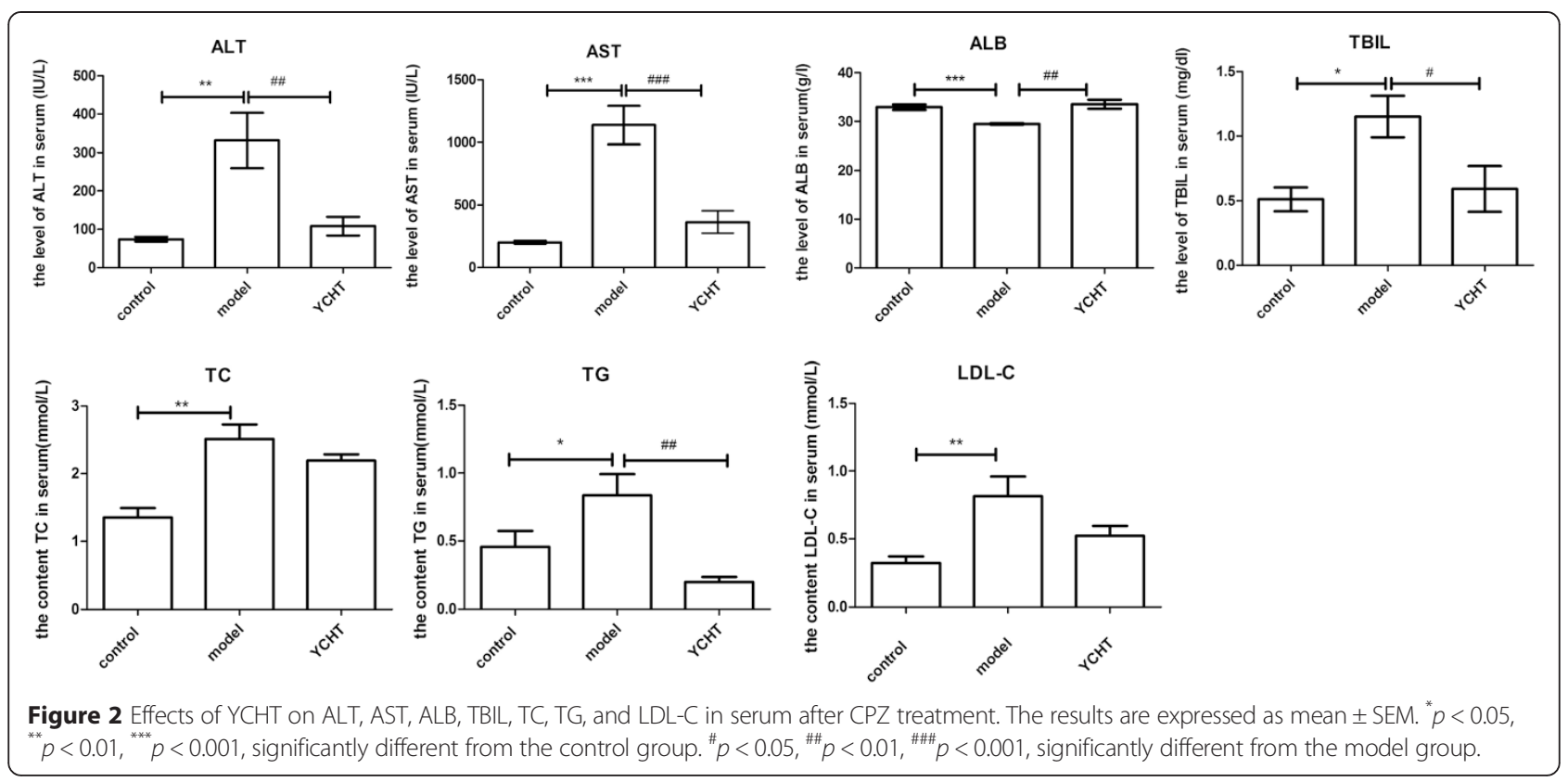

FFAs were exported to SIMCA-P software for the multivariate statistical analysis in the form of principal component analysis and partial least squares discriminant analysis (PLS-DA). The parameters adopted to evaluate the model quality included $R^{2}$ and $Q^{2}$. The $R^{2}$ values indicated the explained variation, and the $\mathrm{Q}^{2}$ values indicated the predictive ability. Figure 4 displays the result of PLS-DA model, which shows clusters and separations from the control, model, and YCHT groups, indicating that CPZ injection affected the metabolism of BAs and FFAs. The group pretreated with YCHT was located between the model and control groups, indicating that YCHT gradually adjusted the pathological condition to physiological condition. Combined the selected variables with VIP values larger than 1 and the significant statistical analysis, $\alpha$-MCA, $\beta$-MCA, CA, DCA, TCDCA, TDCA, THDCA, GCA, HDCA,UDCA,C18:1n9, C18:2n6, and $\mathrm{C} 20: 5 \mathrm{n} 3$ were recognized as the most important parameters for elucidating the cholestasis process and evaluating the effect of YCHT on CPZ-induced cholestatic liver injury.
BAs are recognized as regulatory molecules that are involved in major metabolic progress and show dynamic variances. In this study, the quantitative results and variation tendencies of serum BA profiling are shown in Figure 5 and Table 3, respectively. BA concentration significantly varied in the three groups. Increasing primary $\mathrm{BA}$ concentrations were detected in rats with $\mathrm{CPZ}$ induced cholestatic liver injury. However, the serum concentration of secondary BAs decreased in rats with $\mathrm{CPZ}$-induced cholestatic liver injury compared with that in the control group. Compared with the control group, increasing $\alpha$-MCA, $\beta$-MCA, $\omega$-MCA, CA, CDCA, and corresponding conjugated $\mathrm{BAs}$ were observed in the model group, whereas secondary BAs and corresponding conjugated BAs decreased, except for DCA. The groups pretreated with YCHT showed reversed effect.

In addition, the FFA concentrations in the serum of the control, model, and treated groups were quantified by the optimal GC-MS conditions described in this study. These FFA concentrations in the three groups were compared by one-way ANOVA with LSD post hoc analysis. Despite

Table 2 Effect of YCHT on the biochemical parameters of serum

\begin{tabular}{|c|c|c|c|c|c|c|c|}
\hline Group & $\begin{array}{l}\text { ALT } \\
\text { (IU/L) }\end{array}$ & $\begin{array}{l}\text { AST } \\
(I U / L)\end{array}$ & $\begin{array}{l}\text { ALB } \\
(I U / L)\end{array}$ & $\begin{array}{l}\text { TBIL } \\
\text { (mg/dl) }\end{array}$ & $\begin{array}{l}\mathrm{TC} \\
(\mathrm{mmol} / \mathrm{L})\end{array}$ & $\begin{array}{l}\text { TG } \\
(\mathrm{mmol} / \mathrm{L})\end{array}$ & $\begin{array}{l}\text { LDL-C } \\
(\mathrm{mmol} / \mathrm{L})\end{array}$ \\
\hline control & $74.17 \pm 6.42$ & 202.3311 .16 & $32.93 \pm 0.55$ & $0.51 \pm 0.09$ & $1.35 \pm 0.14$ & $0.465 \pm 0.12$ & $0.327 \pm 0.05$ \\
\hline model & $331.33 \pm 71.78^{* *}$ & $1138 \pm 153.53^{* * *}$ & $29.5 \pm 0.17^{* *}$ & $1.15 \pm 0.16$ & $2.38 \pm 0.21^{* *}$ & $0.847 \pm 0.16^{*}$ & $0.82 \pm 0.15^{* *}$ \\
\hline YCHT & $108.33 \pm 24.56^{\# \#}$ & $363.83 \pm 89.19^{\# \# \#}$ & $33.57 \pm 0.9^{\# \# \#}$ & $0.59 \pm 0.18$ & $2.32 \pm 0.19$ & $0.2 \pm 0.03^{\# \#}$ & $0.52 \pm 0.07$ \\
\hline
\end{tabular}

The results are expressed as mean \pm SEM. ${ }^{*} p<0.05,{ }^{* *} p<0.01,{ }^{* * *} p<0.001$, significantly different from control group. ${ }^{\#} p<0.05,{ }^{\# \#} p<0.01,{ }^{\# \# \#} p<0.001$, significantly different from model group. 

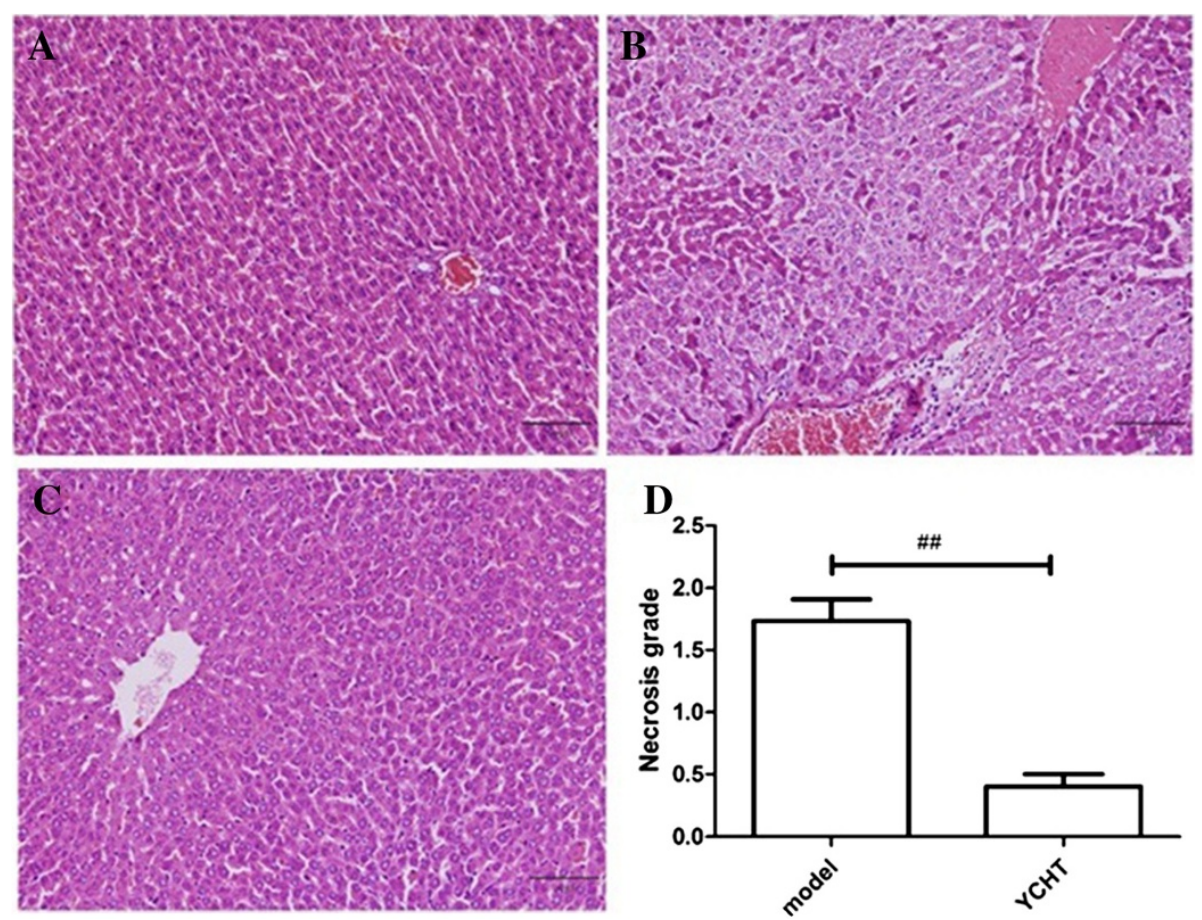

Figure 3 Effect of YCHT on histological changes in the control group (A), model group (B), YCHT group (C) and necrosis grade (D).

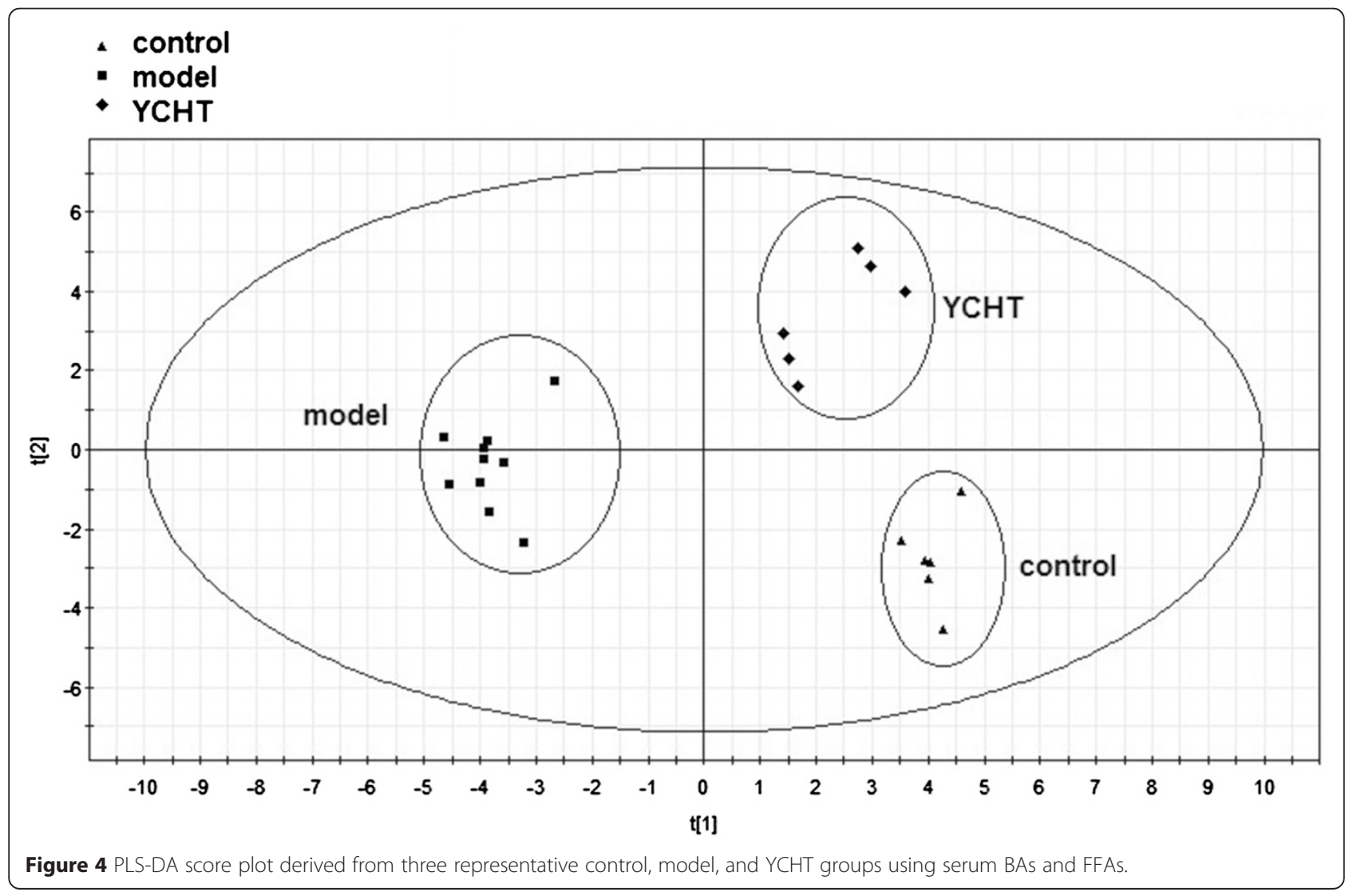




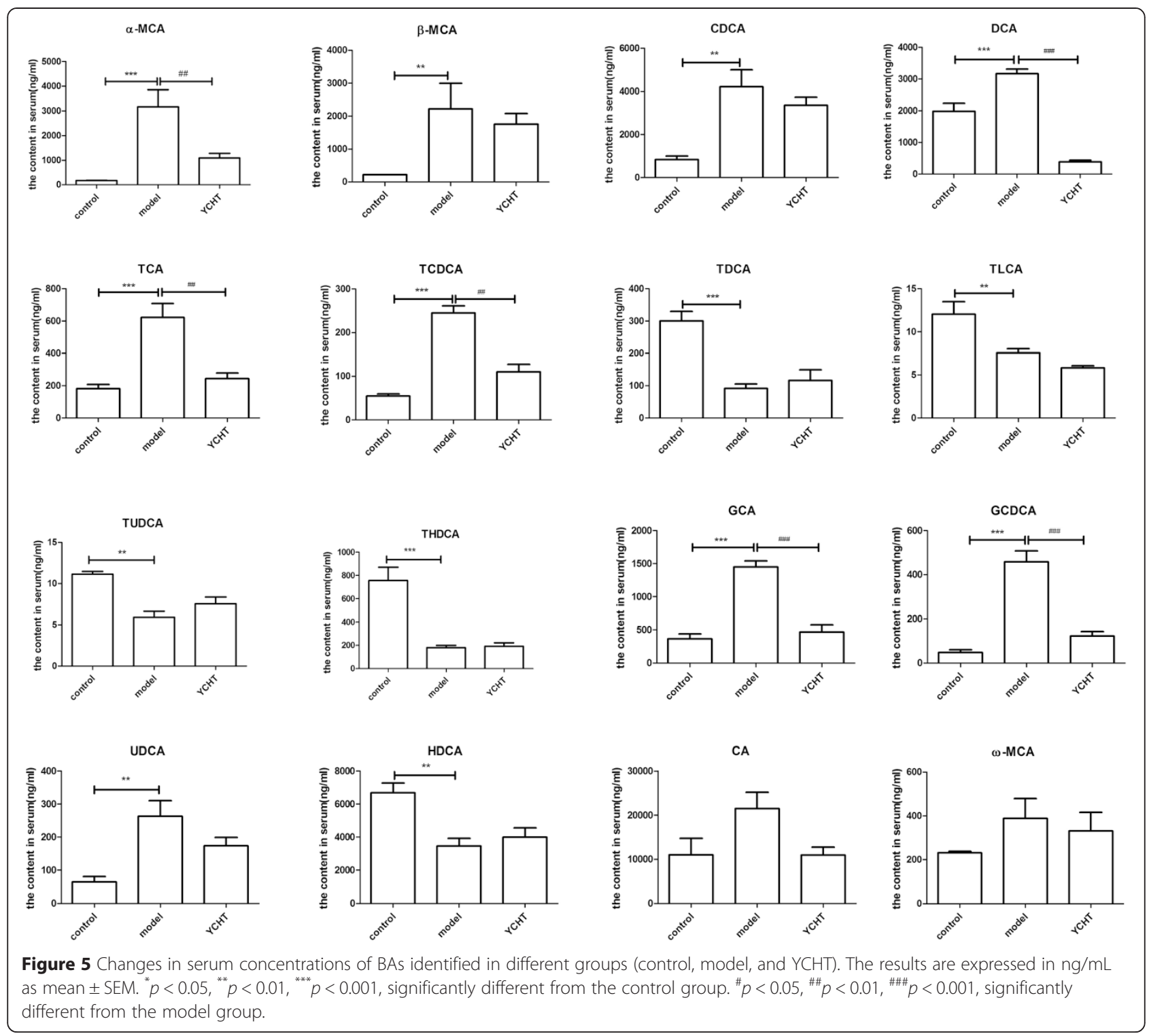

large variations among individuals, dynamic variance was observed for several FFAs. The variations mainly involved $\mathrm{C} 18: 1 \mathrm{n} 9, \mathrm{C} 18: 2 \mathrm{n} 6, \mathrm{C} 18: 3 \mathrm{n} 3, \mathrm{C} 20: 4 \mathrm{n} 6, \mathrm{C} 20: 5 \mathrm{n} 3$, and C22:6n3, as labeled in Figure 6 and summarized in Table 3.

\section{Discussion}

Cholestasis is a common chronic liver disease with variable frequency that confers risks of progression to severe disease and development of end-stage liver disease or specific disease variants. Cholestasis is characterized as a consequence of the disruption of BA homeostasis, the impairment of liver antioxidant defense system or mitochondrial dysfunction. Both hepatocellular functional defects and obstructive lesions of the small bile duct lead to cholestatic liver injury. Cholestasis causes the accumulation of BAs in liver and limit the elimination of
BAs in hepatocytes. Accumulated BAs in hepatocytes result in oxidative stress, promote hepatocyte necrosis, and liver apoptosis. Meanwhile, the mechanism of cholestasis is often associated with hepatocellular transporter expression [32-36]. Previous work reports that the mechanism of CPZ-induced cholestatic liver injury is associated with inhibition of BSEP and MDR3 transcript levels [7]. In this study, the data showed that cholestatic liver injury induced by $\mathrm{CPZ}$ perturbed BA homeostasis, manifested by the elevated hydrophobic BAs $(\alpha, \beta$, $\omega$ MCA, DCA, CDCA, and CA) in serum. The retention of hydrophobic BAs can result in mitochondrial dysfunction by generating ROS, which in turn causes liver injury [37]. However, the group pretreated with YCHT restrained the variance of serum levels of hydrophobic BAs $(\alpha, \beta, \omega$-MCA, DCA, CDCA, and CA). 
Table 3 Effect of YCHT on bile acids (BAs) and free fatty acids (FFAs) metabolism

\begin{tabular}{|c|c|c|c|}
\hline Compound & Control & Model & YCHT \\
\hline CA & $10959.98 \pm 3777.06$ & $21454.91 \pm 3738.66$ & $10936.3 \pm 1774.1$ \\
\hline$a-M C A$ & $175.72 \pm 4.15$ & $3171.48 \pm 690.76^{* * *}$ & $1092.45 \pm 188.1^{\# \#}$ \\
\hline$\beta-M C A$ & $218.17 \pm 5.07$ & $2222.1 \pm 771.15^{* *}$ & $1754.07 \pm 333.24$ \\
\hline$\omega-M C A$ & $231.65 \pm 5.85$ & $388.25 \pm 90.3$ & $331.23 \pm 84.9$ \\
\hline CDCA & $832.91 \pm 157.56$ & $4203.03 \pm 796.24^{* *}$ & $3348.31 \pm 377.81$ \\
\hline DCA & $1979.46 \pm 262.43$ & $3166.18 \pm 144.98^{* * *}$ & $383.96 \pm 54.89^{\# \# \#}$ \\
\hline LCA & $24.46 \pm 2.48$ & $14.07 \pm 1.19^{*}$ & $21.44 \pm 4.16$ \\
\hline UDCA & $65.251 \pm 15.73$ & $262.81 \pm 47.24^{* *}$ & $173.88 \pm 25.20$ \\
\hline HDCA & $6679.97 \pm 598.89$ & $3464.91 \pm 463.26^{* *}$ & $3997.04 \pm 564.22$ \\
\hline TCA & $192.81 \pm 25.91$ & $621.46 \pm 85.46^{* * *}$ & $244.33 \pm 34.33^{\# \#}$ \\
\hline TCDCA & $54.39 \pm 7.79$ & $244.88 \pm 5.17^{* * *}$ & $110.19 \pm 17.09^{\# \#}$ \\
\hline TDCA & $299.94 \pm 29.46$ & $91.63 \pm 13.68^{* * *}$ & $115.99 \pm 39.34$ \\
\hline TLCA & $12.03 \pm 1.45$ & $7.53 \pm 0.49^{* *}$ & $5.81 \pm 0.24$ \\
\hline TUDCA & $11.12 \pm 0.34$ & $5.94 \pm 0.70^{* * *}$ & $7.55 \pm 0.80$ \\
\hline THDCA & $757.34 \pm 112.71$ & $180.02 \pm 19.41^{* * *}$ & $192.5 \pm 28.36$ \\
\hline GCA & $364.43 \pm 72.98$ & $1449.35 \pm 89.25^{* * *}$ & $466.99 \pm 110.79$ \\
\hline GCDCA & $47.42 \pm 12.70$ & $458.01 \pm 49.91^{* * *}$ & $122.86 \pm 12.03^{\# \# \#}$ \\
\hline GDCA & $644.59 \pm 147.35$ & $21.98 \pm 3.8^{* * *}$ & $196.62 \pm 36.89$ \\
\hline GLCA & $17.93 \pm 1.56$ & $13.55 \pm 0.76^{* *}$ & $13.62 \pm 0.71$ \\
\hline C 12:0 & $2.81 \pm 0.021$ & $2.60 \pm 0.048$ & $2.47 \pm 0.03$ \\
\hline C 14:0 & $2.78 \pm 0.12$ & $2.64 \pm 0.08$ & $2.23 \pm 0.08$ \\
\hline C $16: 0$ & $54.81 \pm 2.12$ & $59.43 \pm 2.83$ & $42.74 \pm 2.8$ \\
\hline C16:1n7 & $1.77 \pm 0.05$ & $1.88 \pm 0.08$ & $1.53 \pm 0.06$ \\
\hline C18:0 & $35.84 \pm 1.47$ & $37.49 \pm 2.41$ & $29.23 \pm 2.33$ \\
\hline C18:1n9 & $5.867 \pm 0.11$ & $8.58 \pm 0.75^{* *}$ & $4.30 \pm 0.34^{\# \# \#}$ \\
\hline C18:1n7 & $1.90 \pm 0.89$ & $2.01 \pm 1.68$ & $1.75 \pm 0.73$ \\
\hline C18;2n6 & $12.29 \pm 0.3$ & $18.2 \pm 0.04^{* *}$ & $8.69 \pm 0.03^{\# \# \#}$ \\
\hline C18:3n3 & $1.45 \pm 0.03$ & $1.546 \pm 0.03$ & $1.29 \pm 0.03$ \\
\hline C20:0 & $1.56 \pm 0.05$ & $1.532 \pm 0.03$ & $1.45 \pm 0.01$ \\
\hline C20:3n6 & $1.48 \pm 0.03$ & $1.65 \pm 0.03^{* *}$ & $1.53 \pm 0.01^{\#}$ \\
\hline C20:4n6 & $10.60 \pm 0.50$ & $14.74 \pm 0.61^{* * *}$ & $10.92 \pm 0.77^{\# \# \#}$ \\
\hline$C 20: 5 n 3$ & $2.33 \pm 0.05$ & $2.58 \pm 0.16^{*}$ & $2.08 \pm 0.03^{\# \# \#}$ \\
\hline$C 22: 6 n 3$ & $4.91 \pm 0.21$ & $7.73 \pm 0.67^{* * *}$ & $4.95 \pm 0.37^{\# \# \#}$ \\
\hline
\end{tabular}

The results are expressed in $\mathrm{ng} / \mathrm{mL}$ as mean \pm SEM. ${ }^{*} p<0.05,{ }^{* *} p<0.01,{ }^{* * *} p<0.001$, significantly different from control group. ${ }^{*} p<0.05,{ }^{\# \#} p<0.01, "{ }^{\# \#} p<0.001$, significantly different from model group.

BAs are substrates of BA coenzyme A synthase and BA amino acid transferase by conjugating to amino acids (glycine and taurine) that make them more hydrophilic at acidic $\mathrm{pH}$. These substrates are subsequently impermeable to cell membrane and minimize passive absorption. Similar to drug conjugation, the impermeable properties of the conjugated BAs lead to efficient transportation and detoxification. In this study, the increased contents of serum TCA, GCA, TCDCA, and GCDCA in the model group are in accordance with previous reports $[38,39]$. This result may be explained as the consequence of the liver, which reacts with the adaptive response for limiting the hepatic BA overload. In addition, the elevated level of serum conjugated BAs may be caused by the alteration of transporter protein. The significant increases of serum TCA and GCA in the model group in this study were attributed to the multidrug resistance proteins (Mrp2/Mrp3) that possess high affinity for TCA and GCA. YCHT showed reverse activities on cholestatic liver injury based on the alterations of concentration of conjugated BAs. These observations indicate that YCHT protects the liver from cholestatic liver injury by reducing the size of the total BA pool (data not shown). Basing on this finding, we speculate that the mechanism of the cholestatic liver injury induced by $\mathrm{CPZ}$ and the protection of YCHT may be associated with the regulation of BA metabolism.

FFA, an intracellular signaling sensor of PPAR $\alpha$, participates in lipid metabolism, glucose metabolism, BA metabolism, and inflammation. Evidence demonstrates that the disturbance in lipid homeostasis is causally associated with the pathogenesis and progression of cholangiopathies and biliary fibrosis [21,40]. Previous report suggests that FFAs contribute to significant upregulation of NTCP and Cyp7A1 through the induction of the FXR-SHP pathway [22]. Therefore, this study explored the indicators related to lipid metabolism and discovered that the serum levels of biochemical indicators, including TC, TG, and LDL-C, were increased in the model group. The findings also suggested that CPZ induced the disturbance of lipid metabolism. Moreover, the data based on the metabolic profiling of FFAs were reported in this study, which also indicate the disruption of lipid metabolism in the progression of cholestasis. Pretreatment with YCHT showed the reverse effect in the disruption of lipid metabolism induced by $\mathrm{CPZ}$.

Given the limited availability of drugs to treat hepatobiliary diseases, more anti-cholestasis agents that are safe, effective, and well-characterized are needed. YCHT, a well-known TCM, has an anti-apoptotic property, and is considered a hepatoprotective agent and an antioxidant associated with lipid biosynthesis and peroxidant regulation [26,27,40-44]. Previous reports show that YCHT protects against liver injury with cholestasis in animals having bile duct ligation [25,31]. Lan Shaoyang [45] investigated the mechanism by observing the effect of YCHT on the expression levels of hepatic NTCP in rats in cholestasis and damp-heat syndrome models. A study has proved that YCHT ameliorates concanavalin A-induced hepatitis through its inhibitory action against the production of inflammatory cytokine and its intensive action on the production of anti-inflammatory cytokines [46]. Tzung-Yan Lee stated that YCHT can alleviate hepatic 


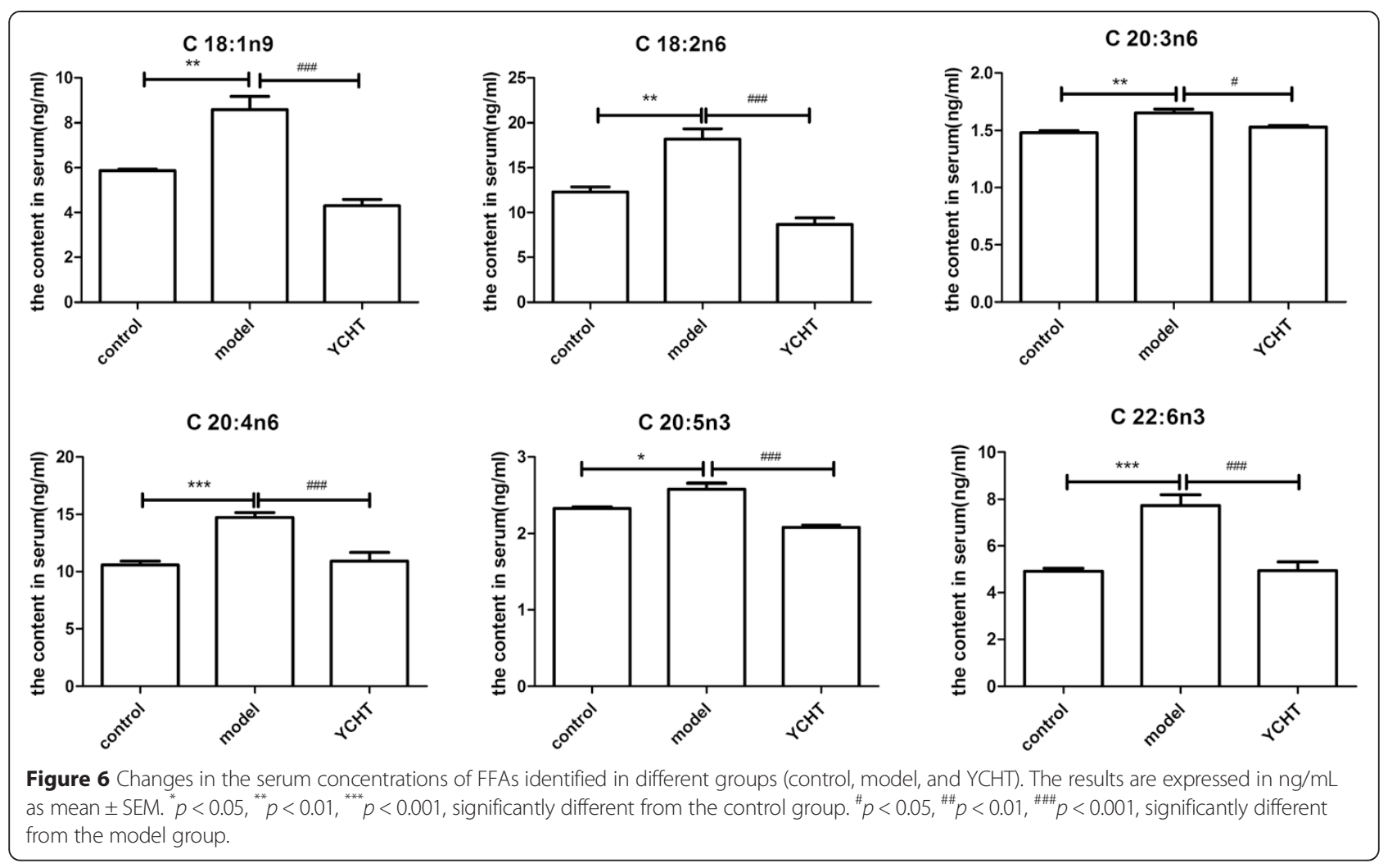

oxidative stress and inhibit fatty acid synthesis in obese mice with steatosis. This finding supports that YCHT contributes to the reduction of serum triglyceride and unsaturated fatty acid concentrations [26], which is consistent with the results of this study. The pretreatment of YCHT decreased CPZ-induced elevation in the serum levels of ALT, AST, TBIL, TC, TG, and LDL-C and up-regulated ALB. Histological examination revealed the suppression of liver injury.

Overall, the combination of LC/GC-MS-based metabolic analysis, animal modeling, and biochemical analysis in this study enabled the characterization of the cholestatic liver injury induced by CPZ in BA and FFA metabolism. This study is the first to use the combination of BAs and FFAs in serum to characterize cholestasis liver injury and evaluate the protective effect of YCHT. The data in this study indicated that YCHT may serve as protective agent against cholestasis liver injury induced by $\mathrm{CPZ}$ in rats. However, evidences for elucidating the mechanism on the protective effect of YCHT against this injury is still lacking. Therefore, further research should focus on the transporters of BAs and FFAs to provide the potential interpretation of the hepatoprotective effects of YCHT.

\section{Conclusions}

The results in this study indicated that YCHT exerted a protective effect on the cholestatic liver injury induced by CPZ. The variance of BA and FFA concentrations can be used to evaluate the cholestatic liver injury caused by $\mathrm{CPZ}$ and the hepatoprotective effect of YCHT.

\section{Competing interests}

The authors declare that they have no financial and personal relationships with other people or organizations that can inappropriately influence their work. And there are no potential conflicts of interest including employment, consultancies, stock ownership, honoraria, paid expert testimony, patent applications and registrations, and grants or other funding.

\section{Authors' contributions}

Conceived and designed the experiments: QY, FY, LY, ZW. Performed the experiments: QY, FY, XT. Analyzed the data: QY, FY, LD, YX, YhX. Wrote the paper: QY, FY, LY. Data acquisition: QY, FY, XT, YX, YhX, LY, ZW. Manuscript revision: $Q Y, F Y, X T, Y X, Y h X, L Y, Z W$. All authors read and approved the final manuscript.

\section{Acknowledgements}

This work is financially supported by the Natural Science Foundations of China (81222053), the Program for New Century Excellent Talents in University (NCET-12-1056) and the Shanghai Municipal Health Bureau Program (XYQ2011061).

\section{Author details}

${ }^{1}$ The Ministry of Education Key Laboratory for Standardization of Chinese Medicines and the State Administration of TCM (SATCM) Key Laboratory for New Resources and Quality Evaluation of Chinese Medicines, Institute of Traditional Chinese Materia Medica, Shanghai University of Traditional Chinese Medicine, 201210 Shanghai, China. ${ }^{2}$ Center for Chinese Medical Therapy and Systems Biology, Shanghai University of Traditional Chinese Medicine, Shanghai 201203, China. ${ }^{3}$ Institute of Traditional Chinese Materia Medica, Shanghai University of Traditional Chinese Medicine, 1200 Cailun Road, Shanghai 201210, China. 
Received: 26 October 2014 Accepted: 20 March 2015

\section{Published online: 16 April 2015}

\section{References}

1. Kremer AE, van Dijk R, Leckie P, Schaap FG, Kuiper EMM, Mettang T, et al Serum autotaxin is increased in pruritus of cholestasis, but not of other origin, and responds to therapeutic interventions. Hepatology. 2012;56:1391-400.

2. De Haan S, Liu X. Chlorpromazine dose for people with schizophrenia. Schizophr Bull. 2009:35:491-2.

3. Derby LE, Gutthann SP, Jick H, Dean AD. Liver disorders in patients receiving chlorpromazine or isoniazid. Pharmacotherapy. 1993;13:353-8.

4. Gandhi A, Guo T, Ghose R. Role of c-Jun N-terminal kinase (JNK) in regulating tumor necrosis factor-alpha (TNF-alpha) mediated increase of acetaminophen (APAP) and chlorpromazine(CPZ) toxicity in murine hepatocytes. J Toxicol Sci. 2010;35:163-73.

5. Gandhi A, Guo T, Shah P, Moorthy B, Ghose R. Chlorpromazine-induced hepatotoxicity during inflammation is mediated by TIRAP-dependent signaling pathway in mice. Toxicol Appl Pharmacol. 2013;266:430-8.

6. Akerboom T, Schneider I, vom Dahl S, Sies H. Cholestasis and changes of portal pressure caused by chlorpromazine in the perfused rat liver. Hepatology. 1991;13:216-21.

7. Antherieu S, Bachour-El Azzi P, Dumont J, Abdel-Razzak Z, Guguen-Guillouzo C, Formenty B, et al. Oxidative stress plays a major role in chlorpromazineinduced cholestasis in human HepaRG cells. Hepatology. 2013;57:1518-29.

8. Sulaiman AA, Al-Shawi NN, Jwaied AH, Mahmood DM, Hussain SA. Protective effect of melatonin against chlorpromazine-induced liver disease in rats. Saudi Med J. 2006;27:1477-82.

9. Uzbekova D, Makarova V, Khvoynitskaya L, Mirgorodskaya L. Protective effects of beehive products and lactulose on chlorpromazine-induced liver damage in rats. Journal of Hepatology. 2002;36:165.

10. Araki H, Peck RC, Lefer AM. Biphasic actions of chlorpromazine and mepacrine on modulation of hepatic cell injury in the perfused cat liver. Arch Int Pharmacodyn Ther. 1981;249:116-25.

11. Li T, Chiang JY. Bile Acid signaling in liver metabolism and diseases. J of Lipids. 2012;2012:1-9.

12. Monte MJ, Marin JJ, Antelo A, Vazquez-Tato J. Bile acids: chemistry, physiology, and pathophysiology. World J Gastroenterol. 2009;15:804-16.

13. Valentini L, Glaser S, Schuetz T, Omar A, Kasim E, Korencke T, et al. Serum bile acids and leptin interact with glucose metabolism in patients with liver cirrhosis. Clin Nutr. 2013;32:122-9.

14. Kamisako T, Ogawa $\mathrm{H}$. Effect of bile duct obstruction on the expression of intestinal mRNA related to cholesterol and bile acid metabolism in the rat. J Gastroenterol Hepatol. 2007;22:125-31.

15. Cray C, Gautier D, Harris DJ, Arheart KL. Changes in clinical enzyme activity and bile acid levels in psittacine birds with altered liver function and disease. J Avian Med Surg. 2008;22:17-24.

16. Crosignani A, Del Puppo M, Longo M, De Fabiani E, Caruso D, Zuin M, et al. Changes in classic and alternative pathways of bile acid synthesis in chronic liver disease. Clin Chim Acta. 2007;382:82-8.

17. Gnewuch C, Liebisch G, Langmann T, Dieplinger B, Mueller T, Haltmayer M et al. Serum bile acid profiling reflects enterohepatic detoxification state and intestinal barrier function in inflammatory bowel disease. World J Gastroenterol. 2009;15:3134-41.

18. Lenicek M, Duricova D, Komarek V, Gabrysova B, Lukas M, Vitek L, et al. Bile acid malabsorption in inflammatory bowel disease: assessment by serum markers. Inflamm Bowel Dis. 2011;17:1322-7.

19. Feldstein AE, Werneburg NW, Canbay A, Guicciardi ME, Bronk SF, Gores GJ, et al. Free fatty acids promote hepatic lipotoxicity by stimulating TNF-alpha expression via a lysosomal pathway. Hepatology. 2014;40:185-94.

20. Malhi H, Bronk SF, Werneburg NW, Gores GJ. Free fatty acids induce JNKdependent hepatocyte lipoapoptosis. J Biol Chem. 2006;281:12093-101.

21. Larter CZ, Yeh MM, Haigh WG, Williams J, Brown S, Lee SP, et al. Hepatic free fatty acids accumulate in experimental steatohepatitis: Role of adaptive pathways. J Hepatol. 2008;48:638-47.

22. Bechmann LP, Kocabayoglu P, Sowa JP, Sydor S, Best J, Berr F, et al. Free fatty acids repress small heterodimer partner (SHP) activation and adiponectin counteracts bile acid-induced liver injury in superobese patients with nonalcoholic steatohepatitis. Hepatology. 2013;57:1394-406.

23. Yang $L$, Xiong A, He Y, Wang Z, Wang C, Hu ZB, et al. Bile acids metabonomics study on the CCl4- and a-Naphthylisothiocyanate-Induced animal models:
Quantitative analysis of 22 bile acids by Ultraperformance Lipid

Chromatography-Mass Spectrometry. Chem Res Toxicol. 2008;21:2280-8.

24. Xiong YH, Xu Y, Yang L, Wang ZT. Gas chromatography-mass spectrometrybased profiling of serum fatty acids in acetaminophen-induced liver injured rats. J Appl Toxicol. 2014;34:149-57.

25. Lee TY, Chang HH, Chen JH, Hsueh ML, Kuo JJ. Herb medicine Yin-ChenHao-Tang ameliorates hepatic fibrosis in bile duct ligation rats. $J$ Ethnopharmacol. 2007;109:318-24.

26. Lee TY, Chang HH, Lo WC, Lin HC. Alleviation of hepatic oxidative stress by Chinese herbal medicine Yin-Chen-Hao-Tang in obese mice with steatosis. Int J Mol Med. 2010;25:837-44.

27. Lee TY, Chang HH, Wu MY, Lin HC. Yin-Chen-Hao-Tang ameliorates obstruction-induced hepatic apoptosis in rats. J Pharm Pharmacol. 2007;59:583-90.

28. Liu C, Liu P, Tao Q. Recipe-syndrome correlation and pathogenesis mechanism of Yinchenhao Decoction in intervening dimethylnitrosamine induced liver cirrhosis progress in rats. Zhongguo Zhong Xi Yi Jie He Za Zhi. 2010;30:845-50.

29. Nomura M, Hida T, Miyamoto K-i, Ohshima T, Hayashi H, Sawanishi H, et al. Synergistic effects of Inchin-ko-to (Yin-Chen-Hao-Tang) and ursodeoxycholic acid on cholestasis caused by a-naphthyl isothiocyanate. Phytother Res. 1995;9:563-6.

30. Wang X-j, Wang P, Sun H, Liu L, Wu Z-m, LV HT, et al. Protective effect and mechanism of Yin-Chen-Hao-Tang (YCHT) against hepatotoxicity induced by ANIT. Acta Chin Med Pharmacol. 2007;35:17-21.

31. Lee TY, Chang HH, Kuo JJ, Shen JJ. Changes of hepatic proteome in bile duct ligated rats with hepatic fibrosis following treatment with Yin-ChenHao-Tang. Int J Mol Med. 2009;23:477-84.

32. Chai J, Luo DL, Wu XP, Wang HZ, He Y, Li Q, et al. Changes of organic anion transporter MRP4 and related nuclear receptors in human obstructive cholestasis. J Gastrointest Surg. 2011;15:996-1004.

33. Soroka CJ, Ballatori N, Boyer JL. Organic solute transporter, OST alpha- OST beta: its role in bile acid transport and cholestasis. Semin Liver Dis 2010;30:178-85

34. Terada T, Chen JR, Ogasawara K, Katsura T, Inui K. Pathophysiological roles of renal organic anion transporter 3 (Oat3) in the cholestasis. Drug Metab Rev. 2008;40:221-2.

35. Blokzijl H, Borght SV, De Vos R, Libbrecht L, Moshage H, Peter L, et al. Cholestasis in acute liver rejection is characterized by intrahepatic cholestasis and adaptive changes in bile salt transporter expression. Hepatology. 2008;48:670a-a.

36. Buis Cl, van der GS, Visser D, Hepkema BG, Sloof MJH, Prote RJ, et al. Evaluation of hepatobiliary transporter polymorphisms in BSEP, MDR-3 and MRP-2 in liver transplant patients with prolonged cholestasis. Liver Transpl. 2006;12:C109-9.

37. Casini A, Ceni E, Salzano R, Biondi P, Parola M, Galli A, et al. Neutrophilderived superoxid anion induces lipid peroxidation and stimulates collagen synthesis in human hepatic stellate cells:role of nitric oxide. Hepatology. 1997;25:361-7.

38. Takikawa H, Otsuka H, Beppu T, Seyama Y, Yamakawa T. Serum concentrations of bile acid glucuronides in hepatobiliary diseases. Digestion. 1983;27:189-95.

39. Meng LJ, Reyes H, Palma J, Hernandez I, Ribalta J, Sjövall J, et al. Effects of ursodeoxycholic acid on conjugated bile acids and progesterone metabolites in serum and urine of patients with intrahepatic cholestasis of pregnancy. J Hepatol. 1997;27:1029-40.

40. Sun H, Zhang A, Yan G, Han Y, Sun W, Ye Y, et al. Proteomics study on the hepatoprotective effects of traditional Chinese medicine formulae Yin-ChenHao -Tang by a combination of two-dimensional polyacrylamide gel electrophoresis and matrix-assisted laser desorption/ionization-time of flight mass spectrometry. J Pharm Biomed Anal. 2013;75:173-9.

41. Wang $X$, Lv H, Sun $H$, Liu L, Yang B, Sun W, et al. Metabolic urinary profiling of alcohol hepatotoxicity and intervention effects of Yin Chen Hao Tang in rats using ultra-performance liquid chromatography/electrospray ionization quadruple time-of- flight mass spectrometry. J Pharm Biomed Anal. 2008:48:1161-8.

42. Cheng HY, Lin LT, Huang HH, Yang CM, Lin CC. Yin Chen Hao Tang, a Chinese prescription, inhibits both herpes simplex virus type-1 and type-2 infections in vitro. Antiviral Res. 2008;77:14-9.

43. Okada K, Shoda J, Kano M, Suzuki S, Ohtake N, Yamamoto M, et al. Inchinkoto, a herbal medicine, and its ingredients dually exert Mrp2- 
mediated choleresis and Nrf2- mediated antioxidative action in rat livers. Am J Physiol Gastrointest Liver Physiol. 2007;292:G1450-63.

44. Kawai K, Yokoyama Y, Kokuryo T, Watanabe K, Kitagawa T, Naqino M, et al. Inchinkoto, an herbal medicine, exerts beneficial effects in the Rat liver under stress with hepatic ischemia-reperfusion and subsequent hepatectomy. Ann Surg. 2010;251:692-700.

45. Lan S, She S, Zhang K. Effect of Yin-Chen-hao decoction on hepatic NTCP expressions in rats with intrahepatic cholestasis and damp-heat syndrome. Traditional Chin Drug Res Clin Pharmacol. 2012;23:279-83.

46. Yamashiki M, Mase A, Arai I, Huang XX, Nobori T, Nishimura A, et al. Effects of the Japanese herbalmedicine 'Inchinko-to' (TJ-135) on concanavalin Ainduced hepatitis in mice. Clin Sci. 2000;99:421-31.

\section{Submit your next manuscript to BioMed Central and take full advantage of:}

- Convenient online submission

- Thorough peer review

- No space constraints or color figure charges

- Immediate publication on acceptance

- Inclusion in PubMed, CAS, Scopus and Google Scholar

- Research which is freely available for redistribution 